\title{
Two tagSNPs rs352493 and rs3760908 within SIRT6 Gene Are Associated with the Severity of Coronary Artery Disease in a Chinese Han Population
}

\author{
Sai-sai Tang, ${ }^{1,2,3}$ Shun Xu, ${ }^{1,2,3}$ Jie Cheng, ${ }^{1,3}$ Meng-yun Cai, ${ }^{1,2,3}$ Lin Chen, ${ }^{1,2,3}$ Li-li Liang, ${ }^{1,2,3}$ \\ Xi-li Yang, ${ }^{4}$ Can Chen, ${ }^{5}$ Xin-guang Liu, ${ }^{1,2,3}$ and Xing-dong Xiong ${ }^{1,2,3}$ \\ ${ }^{1}$ Institute of Aging Research, Guangdong Medical University, Dongguan 523808, China \\ ${ }^{2}$ Institute of Biochemistry \& Molecular Biology, Guangdong Medical University, Zhanjiang 524023, China \\ ${ }^{3}$ Key Laboratory for Medical Molecular Diagnostics of Guangdong Province, Guangdong Medical University, \\ Dongguan 523808, China \\ ${ }^{4}$ Department of Cardiovascular Disease, The First People's Hospital of Foshan, Foshan 528000, China \\ ${ }^{5}$ Department of Cardiovascular Disease, The Affiliated Hospital of Guangdong Medical University, Zhanjiang 524023, China
}

Correspondence should be addressed to Xing-dong Xiong; xiongxingdong@126.com

Received 6 December 2015; Revised 22 February 2016; Accepted 7 March 2016

Academic Editor: Yi-Chia Huang

Copyright (C) 2016 Sai-sai Tang et al. This is an open access article distributed under the Creative Commons Attribution License, which permits unrestricted use, distribution, and reproduction in any medium, provided the original work is properly cited.

SIRT6 has been demonstrated to exert protective effects on endothelial cells and is closely associated with lipid metabolism, glucose metabolism, and obesity, indicating an important role in the pathogenesis and progression of coronary artery disease (CAD). Nonetheless, the biological significance of SIRT6 variants on CAD is far to be elucidated. Here we aimed to investigate the influence of SIRT6 polymorphisms on individual susceptibility and severity of CAD. Multivariate logistic regression analysis exhibited no significant association between these five polymorphisms and CAD risk in the genotype and allele frequencies. However, we found that the rs352493 polymorphism in SIRT6 exhibited a significant effect on the severity of CAD; C allele $\left(\chi^{2}=7.793\right.$, adjusted $P=0.013)$ and the combined CC/CT genotypes $\left(\chi^{2}=5.609\right.$, adjusted $\left.P=0.031\right)$ presented the greater CAD severity. In addition, A allele $\left(\chi^{2}=5.208\right.$, adjusted $\left.P=0.046\right)$ and AA $\left(\chi^{2}=4.842\right.$, adjusted $\left.P=0.054\right)$ of rs3760908 were also associated with greater CAD severity in Chinese subjects. Our data provided the first evidence that SIRT6 tagSNPs rs352493 and rs3760908 play significant roles in the severity of CAD in Chinese Han subjects, which might be useful predictors of the severity of CAD.

\section{Introduction}

Coronary artery disease (CAD) poses the predominant health threat to the public and is the leading cause of death and morbidity worldwide. Previous studies and clinical trials have demonstrated that various environmental factors contribute to the development of CAD, including obesity, diabetes, alcohol intake, tobacco use, hypercholesterolemia, dyslipidemia, and hypertension $[1,2]$. Moreover, apart from these modifiable risk factors, accumulating evidences have established that genetic variants or polymorphisms in candidate genes are closely associated with CAD pathogenesis, which have been estimated to account for $40-60 \%$ of the risk for CAD in epidemiology, family, and twin studies $[3,4]$.
SIRT6, one of the seven members of the $\mathrm{NAD}(+)$ dependent histone deacetylase family of sirtuins, has been considered as one of the most important longevity genes [57]. Transgenic mice overexpressing SIRT6 exhibit a significantly longer life span, while the SIRT6-deficient mice have severe metabolic defects, developed ageing-like phenotypes by 2-3 weeks of age, and eventually died at about 4 weeks $[5,8]$. In addition to the life span regulation function, a growing body of evidences have unraveled that SIRT6 also exerts crucial effects on cell metabolism and DNA damage repair by directly influencing the expression of target genes [9-13]. Among these, the association between SIRT6 and lipid metabolism has been well established. SIRT6 has been reported to serve as a negative regulator of TG synthesis 
and low-density lipoprotein (LDL) cholesterol homeostasis. SIRT6 deficiency resulted in accumulation of TG and LDL cholesterol levels [14-16]. Vice versa, SIRT6-overexpressing mice exhibit a decrease in visceral fat accumulation and improved blood lipid profile [17]. Moreover, the sterolregulatory element binding protein, a key regulator of cholesterol biosynthesis, has been validated as a substrate of SIRT6 [18]. SIRT6 was found to repress the expression of sterolregulatory element binding protein by suppressing its promoter activity and protein cleavage and activating the AMPK signal pathway [19]. Knockout of SIRT6 caused elevated serum cholesterol levels, while ectopic expression of SIRT6 diminished the serum cholesterol levels, thus improving hypercholesterolemia and atherosclerosis [20, 21]. Therefore, it was reasonable to speculate that SIRT6 might exert an important role in CAD pathogenesis and progression.

Single nucleotide polymorphisms (SNPs) are the most frequent genetic variants in the human genome, which have been demonstrated to affect individual susceptibility for a variety of human diseases. Mounting evidences have suggested that SNPs within the candidate genes may potentially contribute to the development of CAD [22-24]. Nonetheless, the genetic causes and underlying molecular mechanisms of these candidate genes for CAD pathogenesis are still far to be elucidated. As SIRT6 plays crucial roles in cellular senescence, genome integrity, and lipid metabolism and improves hypercholesterolemia and atherosclerosis [25], we speculated that the SNPs within the SIRT6 gene might influence the susceptibility and severity of CAD as well. Thus, we herein conducted a case-control study to investigate the associations of five tagSNPs (rs11878868, rs107251, rs352493, rs4807546, and rs3760908) in SIRT6 gene with the risk and severity of CAD. Our results uncovered that rs352493 and rs3760908 polymorphisms within SIRT6 gene were associated with the higher CAD severity in Chinese Han subjects.

\section{Materials and Methods}

2.1. Study Subjects. The study population consisted of 1129 subjects (655 CAD-free controls and 474 CAD patients). All enrolled subjects were recruited from the Affiliated Hospital of Guangdong Medical University (Zhanjiang, China) and the First People's Hospital of Foshan (Foshan, China) from March 2011 to October 2014. All CAD subjects were previously untreated and newly diagnosed. The diagnosis of CAD was confirmed by coronary angiography performed with the Judkins technique. CAD was defined as angiographic evidence of at least one segment of a major epicardial coronary artery with more than $50 \%$ organic stenosis. Patients were divided into three groups (1-vessel, 2-vessel, and 3vessel stenosis) according to the number of significantly stenotic vessels. A total of 655 control individuals were consecutively recruited from the two hospitals for regular physical examinations when CAD subjects were recruited. The unaffected controls were defined to be free of CAD by clinical examination, medical history, electrocardiography, and questionnaires. Subjects with peripheral vascular disease, rheumatic heart disease, congestive heart failure, pulmonary heart disease, hepatic disease, chronic kidney, or any malignancy were excluded from the study. All enrolled subjects were genetically unrelated Han Chinese. The diagnosis of hypertension was established if the individual's systolic blood pressure (SBP) was above $140 \mathrm{~mm} \mathrm{Hg}$ or diastolic blood pressure (DBP) was above $90 \mathrm{~mm} \mathrm{Hg}$ or if the patient was on antihypertensive medication, respectively. Diabetes mellitus was defined as fasting blood glucose (FBG) being above $7.0 \mathrm{mmol} / \mathrm{L}$ or patient being on antidiabetic drug treatment. Subject was defined as smoker if he/she smoked once a day for over 1 year. Dyslipidemia was defined as triglyceride (TG) concentration being $>1.70 \mathrm{mmol} / \mathrm{L}$ or total cholesterol (TC) concentration being $>5.72 \mathrm{mmol} / \mathrm{L}$ or patient being on lipidlowering therapy. This study was approved by the Ethics Committee of the Affiliated Hospital of Guangdong Medical University and the First People's Hospital of Foshan, and informed consent was obtained from all recruited subjects. A structured questionnaire was administered to collect information on demographic data and the risk factors related to CAD.

2.2. Biochemical Analysis. An approximately $2 \mathrm{~mL}$ blood sample was collected from each participant into tubes containing EDTA. The blood sample was centrifuged at $2000 \times \mathrm{g}$ for $15 \mathrm{~min}$ after collection and then stored at $-80^{\circ} \mathrm{C}$ until analysis. Serum concentrations of TC, TG, low-density lipoprotein cholesterol (LDL-C), and high-density lipoprotein cholesterol (HDL-C) were measured enzymatically on a chemistry analyzer (Olympus, Japan). Glucose was measured via the glucose oxidase method (Abbott Laboratories, USA).

2.3. DNA Extraction. Genomic DNA was isolated from peripheral whole blood using TIANamp blood DNA extraction kit (TianGen Biotech, Beijing, China). All DNA samples were dissolved in water and then stored at $-20^{\circ} \mathrm{C}$ until use.

2.4. TagSNP Selection and Genotyping. The Chinese Han population's SNP data of SIRT6 gene $(8,427 \mathrm{bp}, 8$ exons) plus $3 \mathrm{~kb}$ upstream and downstream was obtained in the HapMap data release 27 (http://www.hapmap.org/) [26]. Further analysis of these data was performed utilizing Haploview software version 4.2. Using linkage disequilibrium patterns with $r^{2}>$ 0.8 as a cutoff, five tagSNPs (rs11878868, rs107251, rs352493, rs4807546, and rs3760908) in SIRT6 gene were selected for genotyping. All of the tagSNPs have a MAF (minor allele frequency) $>0.05$ in the HapMap Chinese Han population. The positions of SNPs were shown in Figure 1. These five tagSNPs would capture the information of 7 known SIRT6 SNPs with a MAF > 0.05. The $r^{2}$ information for the five tagSNPs and alleles captured accordingly was exhibited in Table S1 (see Supplementary Material available online at http://dx.doi.org/10.1155/2016/1628041). The potential functions of these SNPs were predicted by a platform freely available online (http://snpinfo.niehs.nih.gov/snpinfo/snpfunc.htm). The haplotypic blocks of the five tagSNPs were estimated by the Haploview software version 4.2 and then the haplotype analysis was done by using the SHEsis software (http://analysis.bio-x.cn/myAnalysis.php) [27]. Six common 


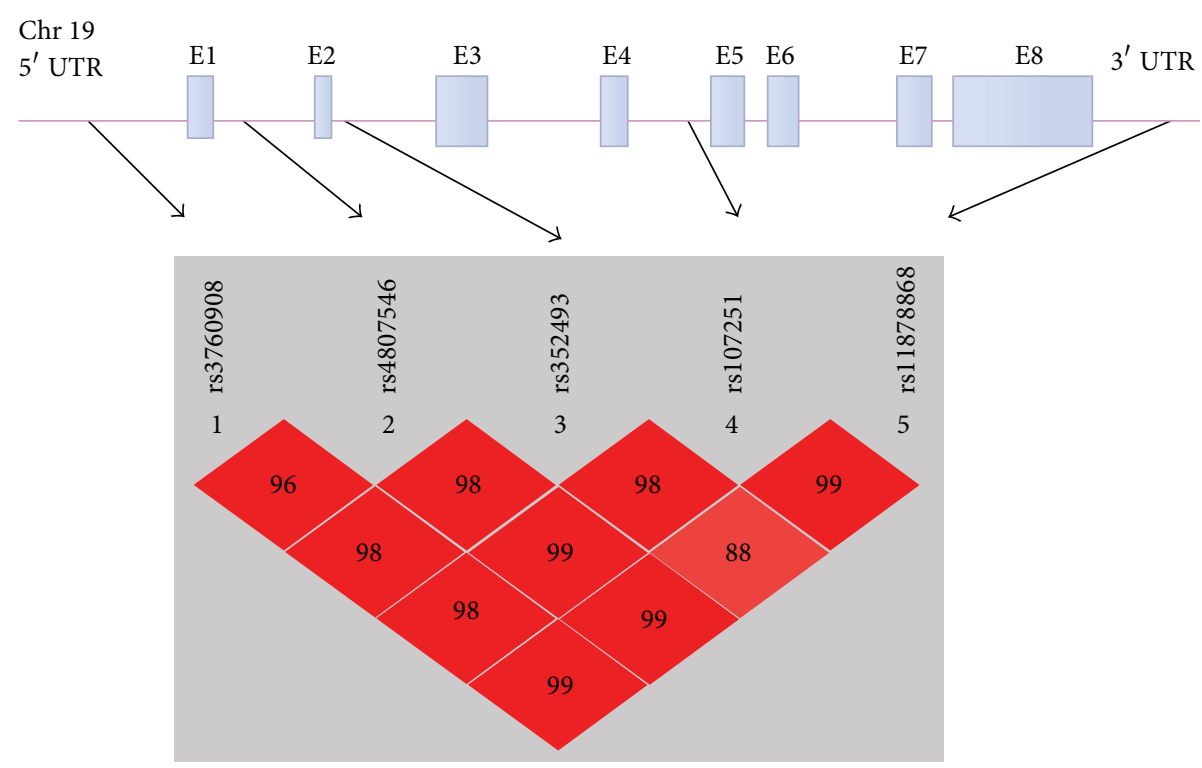

FiguRE 1: Schematic of SIRT6 gene structure and pairwise LD between the five tagSNPs. SIRT6 gene consists of 8 exons (boxes, E1 E8) separated by 7 introns and spans a region of $8,427 \mathrm{bp}$. Filled boxes indicate the coding regions. The arrows indicate the locations of single nucleotide polymorphism. $D^{\prime}$ values are plotted as a graph to show linkage disequilibrium between the five tagSNPs.

haplotypes (frequency $>3 \%$ ) derived from the five tagSNPs accounted for almost $100 \%$ of the haplotype variations. The allelic sequence in the haplotypes is in the following order: rs11878868, rs107251, rs352493, rs4807546, rs3760908. For example, haplotype GCCTA represents rs11878868Grs107251C-rs352493C-rs4807546T-rs3760908A.

Genomic DNA was genotyped by PCR-LDR method (Shanghai Biowing Applied Biotechnology Company). The sequences of probes and primers were summarized in Table S2. The PCR was performed on the GeneAmp PCR system 9600 (Perkin Elmer, Norwalk, CT, USA) in $20 \mu \mathrm{L}$ total volume including $50 \mathrm{ng}$ genomic DNA, 1x PCR buffer, $1 \mathrm{U}$ Taq polymerase, $2 \mathrm{mM}$ dNTPs, $3 \mathrm{mM} \mathrm{MgCl}_{2}$, and $0.5 \mu \mathrm{M}$ primer mix. The cycling parameters were as follows: $95^{\circ} \mathrm{C}$ for $2 \mathrm{~min} ; 40$ cycles at $94^{\circ} \mathrm{C}$ for $90 \mathrm{~s}, 56^{\circ} \mathrm{C}$ for $90 \mathrm{~s}$, and $65^{\circ} \mathrm{C}$ for $30 \mathrm{~s}$; and a final extension step at $65^{\circ} \mathrm{C}$ for $10 \mathrm{~min}$. The ligation reaction for each PCR product was performed with a final volume of $10 \mu \mathrm{L}$ containing $4 \mu \mathrm{L}$ of PCR product, $1 \mu \mathrm{L}$ $1 \mathrm{x}$ buffer, $2 \mathrm{U}$ Taq DNA ligase, and 2 pmol probe mixture. The LDR parameters were as follows: $95^{\circ} \mathrm{C}$ for $2 \mathrm{~min}$ and 40 cycles at $94^{\circ} \mathrm{C}$ for $15 \mathrm{~s}$ and $50^{\circ} \mathrm{C}$ for $25 \mathrm{~s}$. Following the LDR reaction, $1 \mu \mathrm{L} \mathrm{LDR}$ reaction product was mixed with $1 \mu \mathrm{L}$ loading buffer and $1 \mu \mathrm{L}$ ROX. The mixture was then analyzed by the ABI Prism 3730 DNA Sequencer (Applied Biosystems, USA). About $10 \%$ of these samples were randomly selected for repeated genotyping for confirmation, and the results were $100 \%$ concordant.

2.5. Statistical Analysis. The statistical analyses were performed using the SPSS software package (version 21). The haplotype analysis on the polymorphisms was undertaken by using the SHEsis online webserver. All the five SIRT6 tagSNPs were tested for confirmation within Hardy-Weinberg expectations through a goodness-of-fit $\chi^{2}$ test in the control subjects. Qualitative variables were presented as percentages, and quantitative variables were expressed as means \pm standard deviation. Student's $t$-test was used for continuous variables and $\chi^{2}$ test was used for categorical variables in the differences of the demographic characteristics between the CAD and control groups. The association of SIRT6 tagSNPs with CAD severity was evaluated by $\chi^{2}$ test. The association of SIRT6 polymorphisms with the severity of CAD has been determined by logistic regression model with adjustment by age, sex, smoking, drinking, hypertension, diabetes, and hyperlipidemia. Association between the risk for CAD and the tagSNP was evaluated via logistic regression analysis, adjusted by conventional risk factors (age, sex, drinking, smoking, diabetes, hypertension, and hyperlipidemia). $P$ values of less than 0.05 were considered statistically significant for all statistical tests.

\section{Results}

3.1. Characteristics of Study Subjects. The baseline characteristics of the case and control subjects were presented in Table 1. Compared with the controls, a higher rate of patients with CAD were male, smoking and alcohol consumers, with prevalence of hypertension, dyslipidemia, and diabetes, and with higher levels of SBP, FBG, TG, and LDL-C, but lower HDL-C. These data demonstrated that male gender, alcohol intake, smoking, hyperlipidemia, hypertension, and diabetes mellitus were the important risk factors for developing CAD in this study.

3.2. Multivariate Associations of SIRT6 tagSNPs with the Risk of CAD. Five SIRT6 tagSNPs (rs11878868, rs107251, rs352493, rs4807546, and rs3760908) were genotyped in 474 CAD patients and 655 control subjects. The primary information 
TABLE 1: The characteristics of CAD cases and controls.

\begin{tabular}{|c|c|c|c|}
\hline Variable & Controls $(n=655)$ & Cases $(n=474)$ & $P^{\mathrm{a}}$ \\
\hline Age (years) & $61.50 \pm 12.50$ & $63.19 \pm 11.68$ & 0.021 \\
\hline Sex (male) & $379(57.9 \%)$ & $362(76.4 \%)$ & $<0.001^{\mathrm{b}}$ \\
\hline Smoking & $154(23.5 \%)$ & $286(60.3 \%)$ & $<0.001$ \\
\hline Alcohol consumption & $99(15.1 \%)$ & $123(25.9 \%)$ & $<0.001$ \\
\hline Hypertension & $221(33.7 \%)$ & $293(61.8 \%)$ & $<0.001$ \\
\hline Diabetes & $104(15.9 \%)$ & $229(48.3 \%)$ & $<0.001$ \\
\hline Hyperlipidemia & $239(36.5 \%)$ & $314(66.2 \%)$ & $<0.001$ \\
\hline Systolic BP (mm Hg) & $131.83 \pm 19.38$ & $139.70 \pm 19.80$ & $<0.001$ \\
\hline Diastolic BP (mm Hg) & $72.78 \pm 10.63$ & $73.42 \pm 11.24$ & 0.332 \\
\hline FPG $(\mathrm{mM})$ & $5.79 \pm 1.95$ & $6.27 \pm 1.64$ & $<0.001$ \\
\hline Triglycerides (mM) & $1.48 \pm 0.81$ & $1.90 \pm 1.09$ & $<0.001$ \\
\hline Total cholesterol (mM) & $4.59 \pm 1.15$ & $4.67 \pm 1.21$ & 0.266 \\
\hline HDL cholesterol (mM) & $1.39 \pm 0.67$ & $1.24 \pm 0.44$ & $<0.001$ \\
\hline LDL cholesterol (mM) & $2.61 \pm 0.92$ & $2.88 \pm 0.92$ & $<0.001$ \\
\hline
\end{tabular}

${ }^{a}$ Two-sided chi-square test or independent-samples $t$-test.

${ }^{\mathrm{b}} P$ values under 0.05 were indicated in bold font.

TABLE 2: Primary information for tagSNPs in SIRT6 gene.

\begin{tabular}{|c|c|c|c|c|c|}
\hline Genotyped SNPs & rs11878868 & rs107251 & rs352493 & rs4807546 & rs3760908 \\
\hline Chr Pos (genome build 106) & 4173640 & 4176088 & 4180839 & 4182063 & 4184515 \\
\hline Pos in SIRT6 gene & $3^{\prime}$ UTR & Intron 4 & Intron 2 & Intron 1 & $5^{\prime}$ UTR \\
\hline $\mathrm{MAF}^{\mathrm{a}}$ for Chinese $(\mathrm{CHB})$ population in HapMap & 0.110 & 0.310 & 0.240 & 0.390 & 0.430 \\
\hline MAF in controls $(n=655)$ & 0.097 & 0.296 & 0.254 & 0.365 & 0.434 \\
\hline$P$ value for $\mathrm{HWE}^{\mathrm{b}}$ test in controls & 0.411 & 0.773 & 0.785 & 0.839 & 0.093 \\
\hline
\end{tabular}

${ }^{\mathrm{a}} \mathrm{MAF}$ : minor allele frequency.

${ }^{\mathrm{b}}$ HWE: Hardy-Weinberg equilibrium.

for these tagSNPs was shown in Table 2. MAFs for all five tagSNPs in our controls were similar to those for Chinese subjects in HapMap database (Table 2). Genotype frequencies did not deviate from the Hardy-Weinberg equilibrium in the controls (all $P$ values $\geq 0.05$, Table 2 ), providing no evidence of population stratification within the dataset. After adjustment for the risk factors including age, gender, drinking, smoking, hypertension, hyperlipidemia, and diabetes, there was no significant difference between CAD cases and controls in the genotype and allele frequencies in these five tagSNPs (all $P$ values $\geq 0.05$, Table 3 ).

3.3. Association between the Haplotypes of SIRT6 tagSNPs with the Risk of CAD. Haplotype analyses were performed to further investigate the combinational effects of these tagSNPs on CAD risk. As shown in Figure 1, the five tagSNPs were located in one haplotypic block. We thus further assessed the haplotype frequencies of the five tagSNPs between CAD group and controls. Haplotype with frequency over 0.03 will be considered in statistical analysis [28]. Six common haplotypes derived from the five tagSNPs accounted for almost $100 \%$ of the haplotype variations. Among the six common haplotypes, no haplotype was found to be associated with the risk for CAD (Table 4).

3.4. The Association of SIRT6 tagSNPs with the Severity of $C A D$. The severity of CAD was evaluated according to the number of vessels with significant stenosis. To analyze a possible association between SIRT6 tagSNPs and the severity of CAD, patients were grouped into those with 1- and 2vessel disease and 3-vessel disease (50\% luminal obstruction) coronary arteries. The association of SIRT6 tagSNPs with the severity of disease was presented in Table 5. By $\chi^{2}$ test and logistic regression analysis, we found that rs 352493 polymorphism in SIRT6 exhibited a significant effect on the severity of CAD in the Chinese Han population; C allele $\left(\chi^{2}\right.$ $=7.793, P=0.005$, and adjusted $P=0.013)$ and the combined CC/CT genotypes $\left(\chi^{2}=5.609, P=0.018\right.$, and adjusted $P=$ $0.031)$ tended to have greater $\mathrm{CAD}$ severity. In addition, $\mathrm{A}$ allele $\left(\chi^{2}=5.208, P=0.022\right.$, and adjusted $\left.P=0.046\right)$ and AA $\left(\chi^{2}=4.842, P=0.028\right.$, and adjusted $\left.P=0.054\right)$ of rs3760908 also presented the greater CAD severity in the Chinese Han population. 
TABLE 3: Multivariate associations of tagSNPs in SIRT6 gene with the risk of CAD.

\begin{tabular}{|c|c|c|c|c|}
\hline Type & $\begin{array}{c}\text { Controls }(n=655) \\
\text { Number }(\%)\end{array}$ & $\begin{array}{c}\text { Cases }(n=474) \\
\text { Number }(\%)\end{array}$ & OR $(95 \% \mathrm{CI})^{\mathrm{a}}$ & $P^{\mathrm{a}}$ \\
\hline & & rs11878868 & & \\
\hline \multicolumn{5}{|l|}{ Additive } \\
\hline G & $1183(90.3)$ & $856(90.3)$ & 1 & \\
\hline $\mathrm{T}$ & $127(9.7)$ & $92(9.7)$ & $0.97(0.69-1.36)$ & 0.870 \\
\hline \multicolumn{5}{|l|}{ Dominant } \\
\hline $\mathrm{GT}+\mathrm{GG}$ & $647(98.8)$ & $471(99.4)$ & 1 & \\
\hline $\mathrm{TT}$ & $8(1.2)$ & $3(0.6)$ & $0.60(0.14-2.57)$ & 0.487 \\
\hline \multicolumn{5}{|l|}{ Recessive } \\
\hline GG & $536(81.8)$ & $385(81.2)$ & 1 & \\
\hline $\mathrm{GT}+\mathrm{TT}$ & 119 (18.2) & $89(18.8)$ & $1.00(0.69-1.45)$ & 0.993 \\
\hline \multicolumn{5}{|c|}{ rs107251 } \\
\hline \multicolumn{5}{|l|}{ Additive } \\
\hline $\mathrm{T}$ & $388(29.6)$ & $279(29.4)$ & 1 & \\
\hline $\mathrm{C}$ & $922(70.4)$ & $669(70.6)$ & $1.13(0.90-1.41)$ & 0.293 \\
\hline \multicolumn{5}{|l|}{ Dominant } \\
\hline TT & $59(9.0)$ & $37(7.8)$ & 1 & \\
\hline $\mathrm{CC}+\mathrm{CT}$ & $596(91.0)$ & $437(92.2)$ & $1.65(0.98-2.76)$ & 0.060 \\
\hline \multicolumn{5}{|l|}{ Recessive } \\
\hline $\mathrm{CT}+\mathrm{TT}$ & $329(50.2)$ & $242(51.1)$ & 1 & \\
\hline $\mathrm{CC}$ & $326(49.8)$ & $232(48.9)$ & $1.04(0.78-1.39)$ & 0.774 \\
\hline \multicolumn{5}{|c|}{ rs352493 } \\
\hline \multicolumn{5}{|l|}{ Additive } \\
\hline $\mathrm{T}$ & 977 (74.6) & $721(76.1)$ & 1 & \\
\hline $\mathrm{C}$ & $333(25.4)$ & 227 (23.9) & $0.99(0.78-1.25)$ & 0.921 \\
\hline \multicolumn{5}{|l|}{ Dominant } \\
\hline $\mathrm{CC}$ & $41(6.3)$ & $30(6.3)$ & 1 & \\
\hline $\mathrm{CT}+\mathrm{TT}$ & $614(93.7)$ & 444 (93.7) & $0.98(0.54-1.78)$ & 0.944 \\
\hline \multicolumn{5}{|l|}{ Recessive } \\
\hline $\mathrm{CT}+\mathrm{CC}$ & $292(44.6)$ & $197(41.6)$ & 1 & \\
\hline $\mathrm{TT}$ & $363(55.4)$ & $277(58.4)$ & $0.99(0.74-1.32)$ & 0.929 \\
\hline \multirow{2}{*}{\multicolumn{5}{|c|}{ rs4807546 }} \\
\hline & & & & \\
\hline $\mathrm{C}$ & $478(36.5)$ & $352(37.1)$ & 1 & \\
\hline $\mathrm{T}$ & $832(63.5)$ & $596(62.9)$ & $0.94(0.76-1.16)$ & 0.565 \\
\hline \multicolumn{5}{|l|}{ Dominant } \\
\hline $\mathrm{TT}$ & $263(40.2)$ & $189(39.9)$ & 1 & \\
\hline $\mathrm{CT}+\mathrm{CC}$ & $392(59.8)$ & $285(60.1)$ & $0.96(0.72-1.28)$ & 0.763 \\
\hline \multicolumn{5}{|l|}{ Recessive } \\
\hline $\mathrm{CT}+\mathrm{TT}$ & 569 (86.9) & 407 (85.9) & 1 & \\
\hline $\mathrm{CC}$ & $86(13.1)$ & $67(14.1)$ & $0.86(0.57-1.30)$ & 0.478 \\
\hline & & rs3760908 & & \\
\hline \multicolumn{5}{|l|}{ Additive } \\
\hline G & $741(56.6)$ & $562(59.3)$ & 1 & \\
\hline A & $569(43.4)$ & $386(40.7)$ & $0.95(0.77-1.17)$ & 0.616 \\
\hline \multicolumn{5}{|l|}{ Dominant } \\
\hline $\mathrm{AA}$ & $113(17.3)$ & $86(18.1)$ & 1 & \\
\hline $\mathrm{AG}+\mathrm{GG}$ & $542(82.7)$ & $388(81.9)$ & $0.88(0.60-1.29)$ & 0.506 \\
\hline \multicolumn{5}{|l|}{ Recessive } \\
\hline GG & $199(30.4)$ & $174(36.7)$ & 1 & \\
\hline $\mathrm{AG}+\mathrm{AA}$ & $456(69.6)$ & $300(63.3)$ & $0.83(0.61-1.12)$ & 0.212 \\
\hline
\end{tabular}

${ }^{a}$ Adjusted for age, sex, smoking, drinking, hypertension, diabetes, and hyperlipidemia. 
TABLE 4: Association between haplotypes of tagSNPs in SIRT6 gene with the risk of CAD.

\begin{tabular}{|c|c|c|c|c|}
\hline Haplotype $^{\mathrm{a}}$ & $\begin{array}{c}\text { Controls } \\
\text { Number (\%) }\end{array}$ & $\begin{array}{c}\text { Cases } \\
\text { Number (\%) }\end{array}$ & OR (95\% CI) & $P$ \\
\hline Total & $n=655$ & $n=474$ & & \\
\hline GCCTA & $328.69(25.1)$ & $223.42(23.6)$ & $0.93(0.76-1.12)$ & 0.432 \\
\hline GCTCG & $86.43(6.6)$ & $71.83(7.6)$ & $1.17(0.84-1.61)$ & 0.356 \\
\hline GCTTA & $108.25(8.3)$ & $62.54(6.6)$ & $0.79(0.57-1.09)$ & 0.145 \\
\hline GCTTG & $266.76(20.4)$ & $214.82(22.7)$ & $1.15(0.94-1.41)$ & 0.174 \\
\hline GTTCG & $385.25(29.4)$ & $274.28(28.9)$ & $0.98(0.82-1.18)$ & 0.846 \\
\hline TCTTA & $125.02(9.5)$ & $90.91(9.6)$ & $1.01(0.76-1.34)$ & 0.950 \\
\hline
\end{tabular}

${ }^{\mathrm{a}}$ The allelic sequence in the haplotypes is in the following order: rs11878868, rs107251, rs352493, rs4807546, rs3760908.

TABLE 5: SIRT6 tagSNPs in association with diseased vessel $(n=474)$.

\begin{tabular}{|c|c|c|c|c|c|c|}
\hline Type & $\begin{array}{c}1 \mathrm{VD} \text { and } 2 \mathrm{VD}^{\mathrm{a}}(n=292) \\
\text { Number }(\%)\end{array}$ & $\begin{array}{c}3 \mathrm{VD}(n=182) \\
\text { Number }(\%)\end{array}$ & $\chi^{2}$ & $P$ & OR $(95 \% \mathrm{CI})^{\mathrm{c}}$ & $P^{\mathrm{c}}$ \\
\hline \multicolumn{7}{|c|}{ rs11878868 } \\
\hline G & $530(90.8)$ & $326(89.6)$ & \multirow{2}{*}{0.364} & \multirow{2}{*}{0.546} & 1 & \multirow{2}{*}{0.563} \\
\hline $\mathrm{T}$ & $54(9.2)$ & $38(10.4)$ & & & $1.14(0.73-1.80)$ & \\
\hline GG & $240(82.2)$ & 145 (79.7) & \multirow{2}{*}{0.467} & \multirow{2}{*}{0.494} & 1 & \multirow{2}{*}{0.501} \\
\hline $\mathrm{GT}+\mathrm{TT}$ & $52(17.8)$ & $37(20.3)$ & & & $1.18(0.73-1.90)$ & \\
\hline \multicolumn{7}{|c|}{ rs107251 } \\
\hline $\mathrm{C}$ & $405(69.3)$ & $264(72.5)$ & \multirow{2}{*}{1.091} & \multirow{2}{*}{0.296} & 1 & \multirow{2}{*}{0.463} \\
\hline $\mathrm{T}$ & $179(30.7)$ & $100(27.5)$ & & & $0.89(0.66-1.21)$ & \\
\hline $\mathrm{CC}$ & $139(47.6)$ & $93(51.1)$ & \multirow{2}{*}{0.548} & \multirow{2}{*}{0.459} & 1 & \multirow{2}{*}{0.647} \\
\hline $\mathrm{CT}+\mathrm{TT}$ & $153(52.4)$ & $89(48.9)$ & & & $0.92(0.63-1.34)$ & \\
\hline \multicolumn{7}{|c|}{ rs352493 } \\
\hline $\mathrm{T}$ & $462(79.1)$ & $259(71.2)$ & \multirow{2}{*}{7.793} & \multirow{2}{*}{$0.005^{\mathrm{b}}$} & 1 & \multirow{2}{*}{0.013} \\
\hline $\mathrm{C}$ & $122(20.9)$ & $105(28.8)$ & & & $1.47(1.09-2.00)$ & \\
\hline TT & $183(62.7)$ & $94(51.6)$ & \multirow{2}{*}{5.609} & \multirow{2}{*}{0.018} & 1 & \multirow{2}{*}{0.031} \\
\hline $\mathrm{CC}+\mathrm{CT}$ & $109(37.3)$ & $88(48.4)$ & & & $1.52(1.04-2.24)$ & \\
\hline \multicolumn{7}{|c|}{ rs 4807546} \\
\hline $\mathrm{T}$ & $360(61.6)$ & $236(64.8)$ & \multirow{2}{*}{0.978} & \multirow{2}{*}{0.232} & 1 & \multirow{2}{*}{0.443} \\
\hline C & $224(38.4)$ & $128(35.2)$ & & & $0.90(0.68-1.18)$ & \\
\hline $\mathrm{TT}$ & $109(37.3)$ & $80(44.0)$ & \multirow{2}{*}{2.054} & \multirow{2}{*}{0.152} & 1 & \multirow{2}{*}{0.177} \\
\hline $\mathrm{CT}+\mathrm{CC}$ & $183(62.7)$ & $102(56.0)$ & & & $0.77(0.52-1.13)$ & \\
\hline \multicolumn{7}{|c|}{ rs3760908 } \\
\hline A & $221(37.8)$ & $165(45.3)$ & \multirow{2}{*}{5.208} & \multirow{2}{*}{0.022} & 1 & \multirow{2}{*}{0.046} \\
\hline G & $363(62.2)$ & $199(54.7)$ & & & $0.76(0.59-1.00)$ & \\
\hline AA & $44(15.1)$ & $42(23.1)$ & \multirow{2}{*}{4.842} & 0.028 & 1 & 0.054 \\
\hline $\mathrm{GG}+\mathrm{AG}$ & $248(84.9)$ & $140(76.9)$ & & 0.020 & $0.62(0.38-1.01)$ & 0.054 \\
\hline
\end{tabular}

${ }^{a}$ 1VD: 1-vessel disease; 2VD: 2-vessel disease; 3VD: 3-vessel disease. ${ }^{\mathrm{b}} P$ values under 0.05 were indicated in bold font. The $P^{\mathrm{c}}$ values and OR ${ }^{\mathrm{c}}$ were adjusted for age, sex, smoking, drinking, hypertension, diabetes, and hyperlipidemia.

\section{Discussion}

CAD is a complex multifactorial, polygenic disorder that results from both the various environmental factors and the individual's genetic makeup. Recent studies have demonstrated the critical roles of SIRT6 in DNA damage repair, lipid metabolism, and atherosclerosis, providing evidences that SIRT6 may play an important role in the CAD pathogenesis $[20,21]$. Nonetheless, the association of SNPs in SIRT6 gene with CAD risk and severity is largely unknown. In the present study, we proceeded with a genetic association analysis on the five SIRT6 tagSNPs (rs11878868, rs107251, rs352493, rs4807546, and rs3760908) in 474 CAD cases and 655 controls. Our results revealed that $C$ allele of rs 352493 and A allele of rs 3760908 conferred the increased severity of CAD patients in Chinese subjects, though all the five SNPs and haplotypes showed no significant effect on the risk of CAD. Our study suggested that SIRT6 rs352493 and rs3760908 SNPs 
might play roles in the progression of $\mathrm{CAD}$, which could be useful as predictors of the severity of CAD.

The function of SIRT6 on various physiological and pathological processes has been widely studied, and SIRT6 deficiency or dysregulation is closely associated with diverse human diseases including cardiac hypertrophy, adipocyte disorders, and atherosclerosis [18, 29-31]. Nonetheless, the relationship between SIRT6 variants and the risk of these diseases remains largely unknown. Previous study has revealed that T-carriers of rs107251 polymorphism in SIRT6 exhibited an enhanced risk of carotid plaque [32], while individuals with either CC or CT genotype at rs107251 within SIRT6 displayed $>5$-year mean survival advantages compared to TT genotype [33], which is consistent with the protective role of SIRT6. Alternatively, SIRT6 tagSNP rs4807546 showed no significant association with the risk of Parkinson's disease in a Spanish population [34], and the SIRT6 polymorphisms (rs350852, rs7246235, rs107251, and rs350844) were not associated with diabetic nephropathy in a combined metaanalysis as well [35]. Thus, it was not unexpected that the five tagSNPs (rs11878868, rs107251, rs352493, rs4807546, and rs3760908) in this study exhibited no significant association with the risk of CAD.

Multiple variants or polymorphisms within candidate genes have been reported to be associated with the severity of CAD, which is defined by the number of vessels with significant stenosis [36]. CT or TT genotype of rs7903146 polymorphism in TCF7L2 exhibited a higher prevalence and severity of CAD [37]; and the T allele of rs5065 polymorphism in ANP showed a decreased severity of CAD in an Iranian population [38]. We herein presented the evidence that C allele of rs352493 and A allele of rs3760908 polymorphisms conferred the increased severity of CAD patients in the Chinese Han population, further expanding the knowledge of the associations between polymorphisms and the progression of CAD. Nonetheless, the underlying molecular mechanisms of SIRT6 polymorphisms on the severity of CAD still warrant further investigations.

Several limitations in this study still need to be addressed. First, the CAD patients and control subjects enrolled from hospitals may not represent the general population. Nonetheless, the genotype distribution of the control subjects was in Hardy-Weinberg equilibrium. Second, the relatively moderate sample size limited the statistical power of our study. Finally, further studies in different population could help to further verify the significance of the association between the rs352493 and rs3760908 polymorphisms and the severity of CAD. However, our data provided valuable insights into this area.

\section{Conclusion}

In aggregate, our study unraveled that tagSNPs rs352493 and rs3760908 in SIRT6 gene exhibited a significant effect on the severity of CAD in the Chinese Han population, while all the five tagSNPs and haplotypes showed no significant effect on CAD risk. Further studies with diverse ethnic populations and larger sample size are needed to confirm the general validity of our findings.

\section{Disclosure}

Sai-sai Tang and Shun Xu are co-first authors of the paper.

\section{Competing Interests}

The authors declare that they have no competing interests.

\section{Authors' Contributions}

Sai-sai Tang and Shun Xu contributed equally to this work.

\section{Acknowledgments}

The authors thank the First People's Hospital of Foshan and the Affiliated Hospital of Guangdong Medical University, Guangdong Province, China, for their kind assistance in collecting the samples and data. This work was supported by Grants from the National Natural Science Foundation of China (81370456), the Natural Science Foundation of Guangdong Province (2014A030311015 and 2014A030310027), the Medical Scientific Research Foundation of Guangdong Province (A2015288), the Science and Technology Planning Project of Dongguan City (2013108101057), and Scientific Research Foundation of Guangdong Medical University (B2013002).

\section{References}

[1] X.-H. Zhang, Z. L. Lu, and L. Liu, "Coronary heart disease in China," Heart, vol. 94, no. 9, pp. 1126-1131, 2008.

[2] B. P. Prins, V. Lagou, F. W. Asselbergs, H. Snieder, and J. Fu, "Genetics of coronary artery disease: genome-wide association studies and beyond," Atherosclerosis, vol. 225, no. 1, pp. 1-10, 2012.

[3] T. Kangas-Kontio, A. Huotari, H. Ruotsalainen et al., "Genetic and environmental determinants of total and high-molecular weight adiponectin in families with low HDL-cholesterol and early onset coronary heart disease," Atherosclerosis, vol. 210, no. 2, pp. 479-485, 2010.

[4] S. Zdravkovic, A. Wienke, N. L. Pedersen, M. E. Marenberg, A. I. Yashin, and U. De Faire, "Heritability of death from coronary heart disease: a 36-year follow-up of 20966 Swedish twins," Journal of Internal Medicine, vol. 252, no. 3, pp. 247-254, 2002.

[5] R. Mostoslavsky, K. F. Chua, D. B. Lombard et al., "Genomic instability and aging-like phenotype in the absence of mammalian SIRT6," Cell, vol. 124, no. 2, pp. 315-329, 2006.

[6] D. B. Lombard, B. Schwer, F. W. Alt, and R. Mostoslavsky, "SIRT6 in DNA repair, metabolism and ageing," Journal of Internal Medicine, vol. 263, no. 2, pp. 128-141, 2008.

[7] T. L. A. Kawahara, E. Michishita, A. S. Adler et al., "SIRT6 links histone H3 lysine 9 deacetylation to NF- $\kappa$ B-dependent gene expression and organismal life span," Cell, vol.136, no. 1, pp. 62$74,2009$.

[8] Y. Kanfi, S. Naiman, G. Amir et al., "The sirtuin SIRT6 regulates lifespan in male mice," Nature, vol. 483, no. 7388, pp. 218-221, 2012.

[9] R. A. McCord, E. Michishita, T. Hong et al., "SIRT6 stabilizes DNA-dependent protein kinase at chromatin for DNA doublestrand break repair," Aging, vol. 1, no. 1, pp. 109-121, 2009. 
[10] L. Zhong and R. Mostoslavsky, "SIRT6: a master epigenetic gatekeeper of glucose metabolism," Transcription, vol. 1, no. 1, pp. 17-21, 2010.

[11] Z. Mao, C. Hine, X. Tian et al., "SIRT6 promotes DNA repair under stress by activating PARP1," Science, vol. 332, no. 6036, pp. 1443-1446, 2011.

[12] G. Jia, L. Su, S. Singhal, and X. Liu, "Emerging roles of SIRT6 on telomere maintenance, DNA repair, metabolism and mammalian aging," Molecular and Cellular Biochemistry, vol. 364, no. 1-2, pp. 345-350, 2012.

[13] Z. Mao, X. Tian, M. Van Meter, Z. Ke, V. Gorbunova, and A. Seluanov, "Sirtuin 6 (SIRT6) rescues the decline of homologous recombination repair during replicative senescence," Proceedings of the National Academy of Sciences of the United States of America, vol. 109, no. 29, pp. 11800-11805, 2012.

[14] R. Tao, X. Xiong, R. A. DePinho, C.-X. Deng, and X. C. Dong, "FoxO3 transcription factor and Sirt6 deacetylase regulate low density lipoprotein (LDL)-cholesterol homeostasis via control of the proprotein convertase subtilisin/kexin type 9 (Pcsk9) gene expression," Journal of Biological Chemistry, vol. 288, no. 41, pp. 29252-29259, 2013.

[15] H.-S. Kim, C. Xiao, R.-H. Wang et al., "Hepatic-specific disruption of SIRT6 in mice results in fatty liver formation due to enhanced glycolysis and triglyceride synthesis," Cell Metabolism, vol. 12, no. 3, pp. 224-236, 2010.

[16] S. J. Yang, J. M. Choi, E. Chang, S. W. Park, and C.-Y. Park, "Sirt1 and Sirt6 mediate beneficial effects of rosiglitazone on hepatic lipid accumulation," PLoS ONE, vol. 9, no. 8, Article ID e105456, 2014.

[17] Y. Kanfi, V. Peshti, R. Gil et al., "SIRT6 protects against pathological damage caused by diet-induced obesity," Aging Cell, vol. 9, no. 2, pp. 162-173, 2010.

[18] S. Masri, P. Rigor, M. Cervantes et al., "Partitioning circadian transcription by SIRT6 leads to segregated control of cellular metabolism," Cell, vol. 158, no. 3, pp. 659-672, 2014.

[19] S. Elhanati, Y. Kanfi, A. Varvak et al., "Multiple regulatory layers of SREBP1/2 by SIRT6," Cell Reports, vol. 4, no. 5, pp. 905-912, 2013.

[20] R. Stöhr, M. Mavilio, A. Marino et al., "ITCH modulates SIRT6 and SREBP2 to influence lipid metabolism and atherosclerosis in ApoE null mice," Scientific Reports, vol. 5, article 9023, 2015.

[21] R. Tao, X. Xiong, R. A. DePinho, C.-X. Deng, and X. C. Dong, "Hepatic SREBP-2 and cholesterol biosynthesis are regulated by $\mathrm{FoxO}_{3}$ and Sirt6," Journal of Lipid Research, vol. 54, no. 10, pp. 2745-2753, 2013.

[22] X.-D. Xiong, M. Cho, X.-P. Cai et al., "A common variant in premiR-146 is associated with coronary artery disease risk and its mature miRNA expression," Mutation Research/Fundamental and Molecular Mechanisms of Mutagenesis, vol. 761, pp. 15-20, 2014.

[23] J. Cheng, M. Cho, J.-M. Cen et al., "A TagSNP in SIRT1 gene confers susceptibility to myocardial infarction in a Chinese Han population," PLoS ONE, vol. 10, no. 2, Article ID e0115339, 2015.

[24] S. Xu, J. Cheng, Y.-N. Chen et al., “The LRP6 rs2302685 polymorphism is associated with increased risk of myocardial infarction," Lipids in Health and Disease, vol. 13, no. 1, article 94, 2014.

[25] S. Kugel and R. Mostoslavsky, "Chromatin and beyond: the multitasking roles for SIRT6," Trends in Biochemical Sciences, vol. 39, no. 2, pp. 72-81, 2014.
[26] J. C. Barrett, B. Fry, J. Maller, and M. J. Daly, "Haploview: analysis and visualization of LD and haplotype maps," Bioinformatics, vol. 21, no. 2, pp. 263-265, 2005.

[27] Y. Y. Shi and L. He, "SHEsis, a powerful software platform for analyses of linkage disequilibrium, haplotype construction, and genetic association at polymorphism loci," Cell Research, vol. 15, no. 2, pp. 97-98, 2005.

[28] L. Li, Y. Pan, L. Dai, B. Liu, and D. Zhang, "Association of genetic polymorphisms on vascular endothelial growth factor and its receptor genes with susceptibility to coronary heart disease," Medical Science Monitor, vol. 22, pp. 31-40, 2016.

[29] J. H. Duarte, "Osteoarthritis: SIRT6 prevents chondrocyte senescence and DNA damage," Nature Reviews Rheumatology, vol. 11, no. 5, p. 260, 2015.

[30] M. Ailixiding, Z. Aibibula, M. Iwata et al., "Pivotal role of Sirt6 in the crosstalk among ageing, metabolic syndrome and osteoarthritis," Biochemical and Biophysical Research Communications, vol. 466, no. 3, pp. 319-326, 2015.

[31] A. Cardus, A. K. Uryga, G. Walters, and J. D. Erusalimsky, "SIRT6 protects human endothelial cells from DNA damage, telomere dysfunction, and senescence," Cardiovascular Research, vol. 97, no. 3, pp. 571-579, 2013.

[32] C. Dong, D. Della-Morte, L. Wang et al., "Association of the sirtuin and mitochondrial uncoupling protein genes with carotid plaque," PLoS ONE, vol. 6, no. 11, Article ID e27157, 2011.

[33] M. J. TenNapel, C. F. Lynch, T. L. Burns et al., "SIRT6 minor allele genotype is associated with $>5$-year decrease in lifespan in an aged cohort," PLoS ONE, vol. 9, no. 12, Article ID el15616, 2014.

[34] S. Jesús, P. Gómez-Garre, F. Carrillo et al., "Genetic association of sirtuin genes and Parkinson's disease," Journal of Neurology, vol. 260, no. 9, pp. 2237-2241, 2013.

[35] S. Maeda, D. Koya, S.-I. Araki et al., "Association between single nucleotide polymorphisms within genes encoding sirtuin families and diabetic nephropathy in Japanese subjects with type 2 diabetes," Clinical and Experimental Nephrology, vol. 15, no. 3, pp. 381-390, 2011.

[36] A. I. Guney, D. Ergec, D. Kirac et al., "Effects of ACE polymorphisms and other risk factors on the severity of coronary artery disease," Genetics and Molecular Research, vol. 12, no. 4, pp. 6895-6906, 2013.

[37] A. G. P. Sousa, G. F. Marquezine, P. A. Lemos et al., "TCF7L2 polymorphism rs7903146 is associated with coronary artery disease severity and mortality," PLoS ONE, vol. 4, no. 11, Article ID e7697, 2009.

[38] S. Ziaee, S. Kalayinia, M. A. Boroumand et al., "Association between the atrial natriuretic peptide rs5065 gene polymorphism and the presence and severity of coronary artery disease in an Iranian population," Coronary Artery Disease, vol. 25, no. 3, pp. 242-246, 2014. 


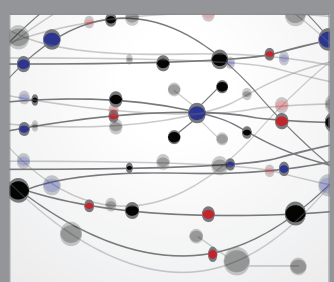

The Scientific World Journal
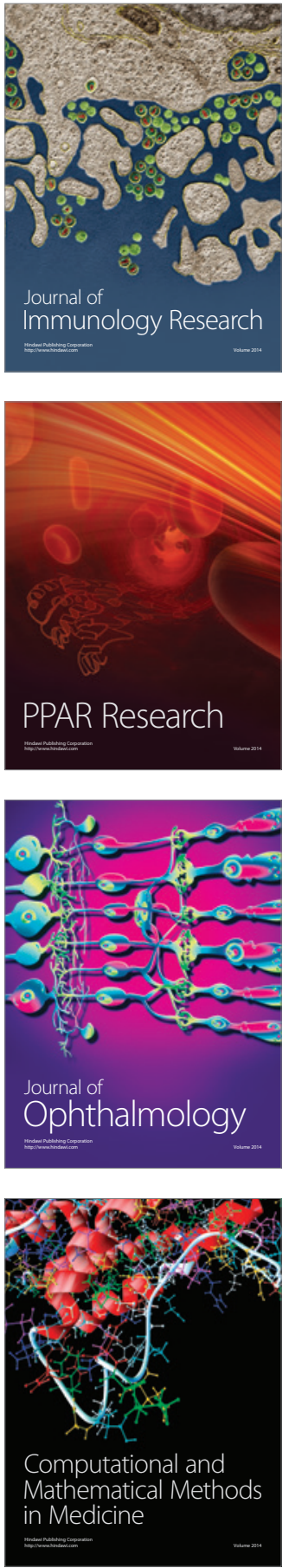

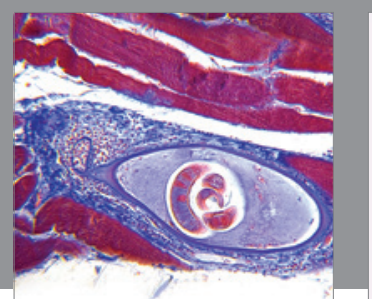

Gastroenterology Research and Practice

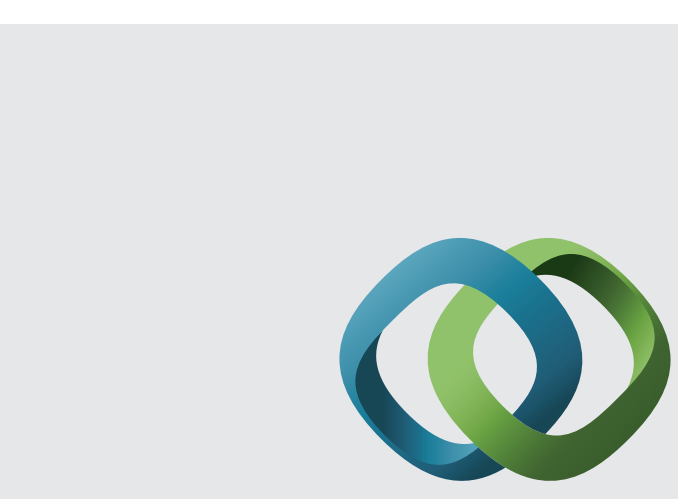

\section{Hindawi}

Submit your manuscripts at

http://www.hindawi.com
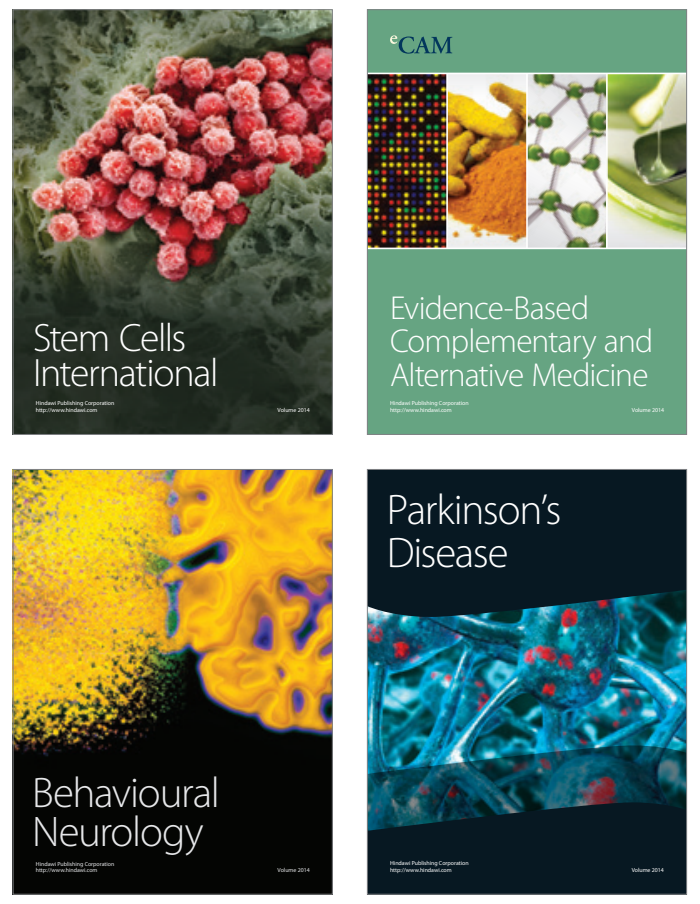
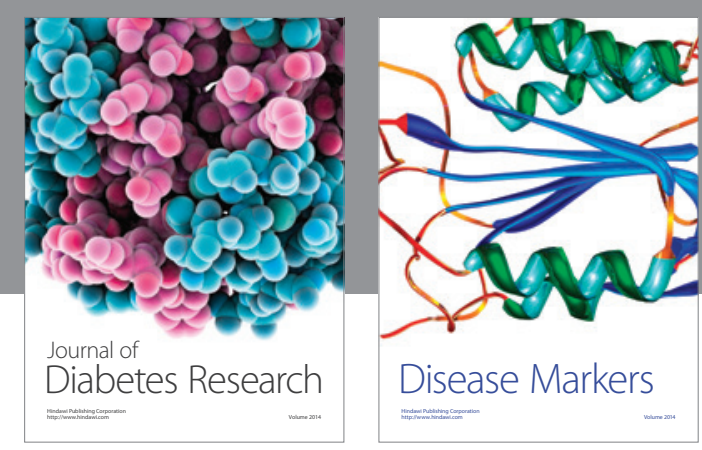

Disease Markers
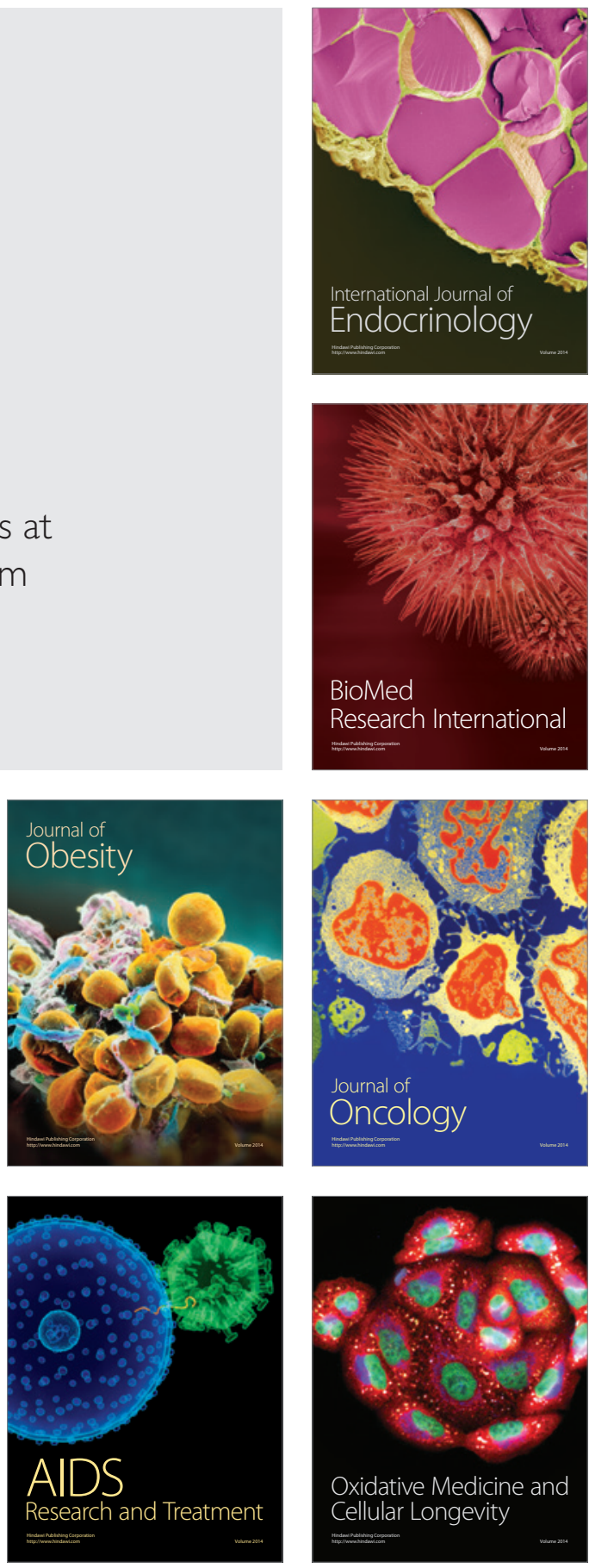